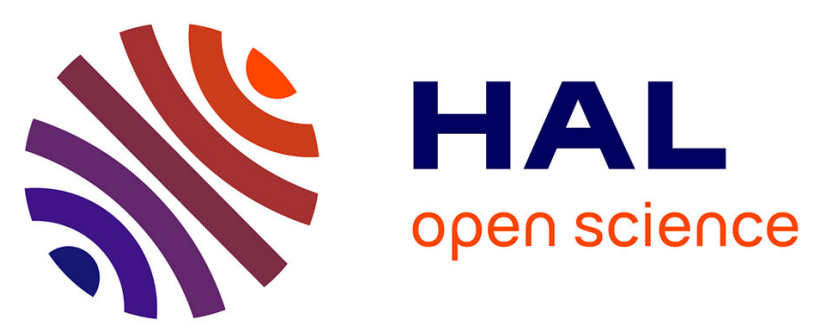

\title{
Computer-based tailored dietary counseling improves the nutrient adequacy of the diet of French pregnant women: a randomized controlled trial
}

Clélia Bianchi, François Mariotti, Anne A. Lluch, Claire Journet, Yaëlle Stehr, Hélène Beaussier, Julien Fournier, Stéphane Dervaux, Dylan Cohen-Tanuggi, Elodie Reulet, et al.

\section{To cite this version:}

Clélia Bianchi, François Mariotti, Anne A. Lluch, Claire Journet, Yaëlle Stehr, et al.. Computerbased tailored dietary counseling improves the nutrient adequacy of the diet of French pregnant women: a randomized controlled trial. British Journal of Nutrition, 2020, 123 (2), pp.220-231. 10.1017/S0007114519002617 . hal-02366520

\section{HAL Id: hal-02366520 \\ https://hal.science/hal-02366520}

Submitted on 20 Sep 2020

HAL is a multi-disciplinary open access archive for the deposit and dissemination of scientific research documents, whether they are published or not. The documents may come from teaching and research institutions in France or abroad, or from public or private research centers.
L'archive ouverte pluridisciplinaire HAL, est destinée au dépôt et à la diffusion de documents scientifiques de niveau recherche, publiés ou non, émanant des établissements d'enseignement et de recherche français ou étrangers, des laboratoires publics ou privés. 
Computer-based tailored dietary counseling improves the nutrient adequacy of the diet of French pregnant women: a randomized controlled trial

Clélia M. Bianchi ${ }^{1}$, François Mariotti ${ }^{*}$, Anne Lluch ${ }^{2}$, Claire Journet ${ }^{1}$, Yaëlle Stehr ${ }^{3}$, Hélène Beaussier ${ }^{4}$, Julien Fournier ${ }^{4}$, Stéphane Dervaux ${ }^{5}$, Dylan Cohen-Tanuggi ${ }^{5}$, Elodie Reulet ${ }^{1}$, Eric O. Verger ${ }^{6}$, Elie Azria ${ }^{3,7}$, Jean-François Huneau ${ }^{1}$

${ }^{1}$ UMR PNCA, AgroParisTech, INRA, Université Paris-Saclay, 75005, Paris, France.

${ }^{2}$ Global Nutrition Department, Danone Nutricia Research, Centre Daniel Carasso, RD 128, 91737 Palaiseau Cedex, France.

${ }^{3}$ Notre Dame de Bon Secours Maternity Unit, Groupe Hospitalier Paris Saint-Joseph, 75014, Paris, France.

${ }^{4}$ Clinical Research Center, Groupe Hospitalier Paris Saint-Joseph, 75014, Paris, France

${ }^{5}$ UMR MIA-Paris, AgroParisTech, INRA, Université Paris-Saclay, 75005, Paris, France.

${ }^{6}$ NUTRIPASS, IRD, Université de Montpellier, SupAgro, 34000 Montpellier, France.

${ }^{7}$ UMR1153 - Obstetrical, Perinatal and Pediatric Epidemiology (EPOPé research team), DHU Risks in Pregnancy, Paris Descartes University - INSERM, 75014, Paris, France.

*Author responsible for correspondence and to whom requests for reprints should be addressed: Prof. François Mariotti; Mailing address: AgroParisTech, UMR 914 PNCA, 16 rue Claude Bernard, F-75005, Paris, France; email: francois.mariotti@agroparistech.fr;

This peer-reviewed article has been accepted for publication but not yet copyedited or typeset, and so may be subject to change during the production process. The article is considered published and may be cited using its DOI

$10.1017 / \mathrm{S} 0007114519002617$

The British Journal of Nutrition is published by Cambridge University Press on behalf of The Nutrition Society 
Shortened version of the title: Tailored dietary counseling in pregnancy.

Keywords: tailored, dietary counseling, pregnancy, nutrient adequacy, behavior change techniques.

\begin{abstract}
During pregnancy, mothers-to-be should adapt their diet to meet increases in nutrient requirements. Pregnant women appear to be keener to adopt healthier diets, but not always successful.

The objective of this study was to determine whether a guided, stepwise and tailored dietary counseling program, designed using an optimization algorithm, could improve the nutrient adequacy of the diet of pregnant women, beyond generic guidelines.

80 pregnant women who attended Notre-Dame-de-Bon-Secours Maternity Clinic were randomly allocated to the control or intervention arms. Dietary data were obtained twice from an online 3-day dietary record. The nutrient adequacy of the diet was calculated using the PANDiet score, a 100point diet quality index adapted to the specific nutrient requirements for pregnancy. Women were supplied with generic dietary guidelines in a reference booklet. In the intervention arm, they also received nine sets of tailored dietary advice identified by an optimization algorithm as best improving their PANDiet score.
\end{abstract}

78 pregnant women completed the 12-week dietary follow-up. Initial PANDiet scores were similar in the control and intervention arms $(60.4 \pm 7.3$ vs $60.3 \pm 7.3, P=0.92)$. The PANDiet score increased in the intervention arm $(+3.6 \pm 9.3, P=0.02)$ but not in the control arm $(-0.3 \pm 7.3, P=0.77)$, and these changes differed between arms $(P=0.04)$. In the intervention arm, there were improvements in the probabilities of adequacy for ALA, thiamin, folate and cholesterol intakes $(P<0.05)$.

Tailored dietary counseling using a computer-based algorithm is more effective than generic dietary counseling alone in improving the nutrient adequacy of the diet of French women in midpregnancy. 


\section{Introduction}

In recent decades, more evidence has become available in favor of the Developmental Origin of Health and Disease (DOHaD) paradigm (1-3). Indeed, good maternal nutrition, defined by meeting the additional requirements of pregnancy without excessive intakes of unfavorable nutrients, is critical to both the mother's health and well-being and also to that of her child in utero and later in childhood and even adult life (4,5). In developed countries, inadequate intakes of both macro- and micronutrients have been observed during pregnancy $(6,7)$, with high intakes of fat, saturated fats (SFA) (6) and sugars (8), low intakes of fiber and polyunsaturated fats (PUFA) (6), the n-6:n-3 ratio of which is inadequate $(9,10)$. Intakes of vitamin and minerals identified as critical during pregnancy are also of concern, with inadequate intakes reported for vitamin D, folate, iron (7) and iodine $(11,12)$. In France, the few studies that have assessed the nutrient intakes of pregnant women have revealed the same trends, with inadequate intakes of fat, SFA (13), dietary fiber, vitamin D (14), folate $(14,15)$, iron $(15)$ and iodine $(15,16)$ and an inadequate PUFA profile $(10,13)$.

As demonstrated by many qualitative studies, pregnancy is accompanied by a rise in nutrition awareness (17) associated with more nutrition-related information seeking practices (18) and the adoption of healthier diets (19-21). However, despite their motivation to change their diets, pregnant women are not always successful and find themselves lost in the mass of confusing information provided on nutrition-related issues and are looking to receive credible and trustworthy dietary advice (21-23). This places pregnancy as a window of opportunity to implement dietary interventions by means of counseling. An increasing number of lifestyle interventions using dietary counseling and targeting pregnant women have thus been designed and have reported benefits regarding the consumption of fruit and vegetables $(24,25)$, intakes of SFA, calcium, potassium, vitamins $\mathrm{A}$ and $\mathrm{C}$, riboflavin and folate (24) or the correct contribution of macronutrients to energy intake (26). Furthermore, lifestyle interventions providing dietary counseling have also been shown to be beneficial to maternal and pregnancy outcomes such as weight gain $(27,28)$, systolic and diastolic blood pressures (29) and the incidence of caesarian deliveries (27), although no clear effects of dietary counseling have been observed with respect to neonatal outcomes (30). Lifestyle interventions and the methods employed to deliver dietary counseling may vary. During the studies, most of these interventions were not tailored but individualized, meaning that a dietitian interacted with the woman in order to provide generic dietary recommendations and/or meal plans. However, nutrition interventions using a computer-based algorithm to provide tailored dietary counseling proved more efficient in improving the diet of individuals, while also reducing costs (31-33). Tailoring implied selecting the most appropriate characteristics of individuals to target the advice, using behavior change techniques adapted to the target population and selecting the best computer- 
based device for data collection and advice provision (32). Our group recently developed a computer-based tailored dietary counseling approach for pregnant women, based on improving the nutrient adequacy of their individual diets, as assessed by the PANDiet score $(34,35)$. The acceptability of this approach was evaluated in French pregnant women and the results were used to refine it (35-37). However, no computer-based tailored dietary intervention which takes account of the global nutrient adequacy of the diet has been reported to date in pregnant women with a normal pre-pregnancy BMI (Body Mass Index). The objective of this study was therefore to determine whether a guided, stepwise and tailored dietary counseling program, based on an optimization algorithm, could better improve the nutrient adequacy of the diet of pregnant women than an approach based solely on generic guidelines.

\section{Methods}

\section{Study design and ethics}

The study was a 12-week, two-arm, single-blind, randomized, controled clinical trial (Figure 1). The study was registered with the French National Agency for Medicines and Health Products Safety (Agence Nationale de Sécurité du Médicament et des Produits de Santé) with the identification number: ID RCB 2016-A00853648, under the administrative supervision of the French Ministry of Health. The study was also approved by two French Ethics Committees (Comité de Protection des Personnes (CPP) Île-de-France II (identification number: 2016-07-02 MS1 SC) and Comité Consultatif sur le Traitement de l'Information en matière de Recherche dans le domaine de la Santé (CCTIRS) (identification number: 16-259)). The trial was registered at clinicaltrials.gov as NCT03084627. Written informed consent was obtained from all subjects before any data collection.

For inclusions, 186 women were approached when attending their first appointment with the midwife in the maternity clinic or by means of flyers and posters displayed in the clinic's waiting rooms. On the day of their inclusion, all participants filled three questionnaires. The first focused on social and demographic features, the second assessed some pregnancy characteristics (gestational diabetes, number of fetus and complications during current and previous pregnancies), and the third evaluated basic characteristics of their lifestyle (including diet). The baseline characteristics of participants are presented in Table 1. The participants were randomized within two weeks of their inclusion; during weeks 2 and 3 (initial assessment), their diet was evaluated from a 3-day online dietary record (see "Dietary assessment" section). At the beginning of week 5 , the participants attended their first dietetic appointment with the dietitian, which lasted between 30 and 45 minutes. Details about the content of dietetic appointments for the control and intervention arms are available 
in the "Dietetic appointment content" section. At the beginning of weeks 7 and 9, all participants were contacted by phone by the dietitian regarding their second and third dietetic appointments. During weeks 11 and 12 (final assessment), their diet was evaluated as during weeks 2 and 3. After completing their last diet record, the participants filled two online questionnaires about changes to their dietary habits during pregnancy and the dietary counseling they received during the study. The final questionnaire differed between the control and intervention arms.

The primary outcome measure of the study was differences in the PANDiet score between the initial and final dietary assessments. The secondary outcome measures of the study were changes in the values of the items composing the PANDiet score (adequacy and moderation subscores and probabilities of adequacy for nutrient intakes - see "Nutrient adequacy assessment" section), the frequency with which participants read the booklet in both arms and implementation of the advice they had received (number and frequency) in the intervention arm only.

\section{Participants}

All participants were attending Notre-Dame de Bon Secours Maternity Clinic (Hôpital Paris SaintJoseph, Paris, France) for their antenatal care. The inclusion criteria were as follows: female, pregnant, [10;24[ weeks of amenorrhea, [18;40[ years old, BMI (in kg/m²) of [18.5-25[, fluent in speaking, reading and writing French, an opportunity for daily internet access, a valid email and phone number and benefiting from French national health insurance. Non-inclusion criteria were as follows: multiple pregnancy, at-risk pregnancy (according to the clinic's classification), history of gestational diabetes mellitus (for multiparous women), health conditions requiring a specific diet, vegan diets, or allergies to one or more of the major allergens listed in Regulation $n^{\circ} 1169 / 2011$ of the European Parliament and of the Council. Exclusion criteria included a diagnosis of gestational diabetes during the study period (12 weeks), a diagnosis of pregnancy complications requiring modifications to the diet, or spontaneous abortion. The participants were randomly allocated to two groups in blocks of twelve that were balanced as a function of age, BMI, parity (percentage of primiparous women in each arm) and household income level, using a mathematical method to minimize differences between the arms. The participants were blinded to the existence of two different dietary counseling approaches. All participants were compensated for their trips to the clinic and the time they spent completing the study with a maximum of $400 €$ for complete participation. A diagram of subject flow according to CONSORT guidelines is presented in Figure 2. 


\section{Dietary assessments}

Dietary data were recorded online using Dietlib' (MyGoodLife, Paris, France), which is a professional tool used by French dietitians for patient care that complies with the requirements of the French National Health Authority and the French Association of Dietitians and Nutritionists. The software included a 3-day dietary record using a closed food list (6,287 food items). Portion sizes were estimated using colored images from a national food portion guidebook (38), household measures (e.g. one teaspoon) or commercial servings (e.g. a standard yogurt).

Three non-consecutive days, including two weekdays and one weekend day, were randomly selected for the initial (weeks 2 and 3) and final (weeks 11 and 12) dietary assessments. The participants were informed of the dates for their diet recording periods at least 7 days beforehand. Before each day of recording, an electronic reminder was sent to the participant. Each day of the record was checked by the dietitian within $48 \mathrm{~h}$ of its completion; if any inconsistencies were seen, the participant was contacted by email. Based on these 3-day records, the Energy Intake Excluding Alcohol (EIEA) to Basal Metabolic Rate ratio (determined according to Black et al. (39)) of the participants was calculated. A ratio below 1 was considered to be a potential indicator of underreporting and the participant was contacted and asked to check her dietary records. This contact did not aim at increasing the ratio. If required, the dietary record could slightly be updated.

The nutrient values used to calculate nutrient intake came from the French Food composition database [CIQUAL 2013, (40)]. In exceptional cases, participants might have declared a food item for which no correspondence with the CIQUAL database could be made, so the nutrient values were those provided by the retailer and already included in the software database. For each participant, the mean intakes calculated included weighting for the day of the week (weekday or weekend day) with respect to the following nutrients: alcohol, protein, total carbohydrate, starch, sugars, added sugars, free sugars, total fat, polyunsaturated fatty acids, alpha-linolenic acid (ALA), linoleic acid (LA), eicosapentaenoic acid (EPA), docohexaenoic acid (DHA), monounsatured fatty acids, saturated fatty acids, cholesterol, dietary fiber, vitamin A, thiamin, riboflavin, niacin, pantothenic acid, vitamin B6, folate, vitamins B12, C, D and E, calcium, iodine, iron, magnesium, phosphorus, potassium, selenium and zinc.

\section{Assessment of nutrient adequacy}

The nutrient adequacy of each participant's diet was assessed from the initial and final dietary records using the PANDiet diet quality index, previously adapted to the specific requirements for the third trimester of pregnancy (34). Briefly, the PANDiet aims to measure the overall diet quality of an individual by combining the probabilities of having an adequate intake of nutrients. The PANDiet is a 100-point score that results from averaging two sub-scores, the Adequacy sub-score 
(Adeq-S) and the Moderation sub-score (Mod-S); the higher the PANDiet score, the better the nutrient adequacy. Each sub-score is composed of probabilities of adequacy for nutrients (27 for Adeq-S: protein, total carbohydrate, dietary fiber, total fat, LA, ALA, DHA, EPA+DHA, vitamin A, thiamin, riboflavin, niacin, pantothenic acid, vitamin B6, folate, vitamin B12, vitamin C, vitamin D, vitamin E, calcium, iodine, iron, magnesium, phosphorus, potassium, selenium and zinc, and seven for Mod-S: protein, total carbohydrate, free sugars, total fat, saturated fatty acids, cholesterol and sodium), with a further 14 potential penalties for exceeding the tolerable upper intake limits (retinol, niacin, vitamin B6, folate, vitamin $\mathrm{C}$, vitamin $\mathrm{D}$, vitamin $\mathrm{E}$, calcium, iodine, iron, magnesium, phosphorus, selenium and zinc) which are added to Mod-S. Because DHA intakes are considered in the probabilities of adequacy for both DHA and EPA+DHA intakes, each one was weighted by 0.5 in the final score, resulting in an Adeq-S with 26 probabilities of adequacy for nutrient intakes. The dietary reference values used to calculate PANDiet were mostly those issued by the French Agency for Food, Environmental and Occupational Health (Agence Nationale de Sécurité Sanitaire de l'alimentation, de l'environnement et du travail, ANSES) $(34,41)$.

\section{Content of dietetic appointments}

\section{Control arm}

During the first dietetic appointment, participants in the control arm received generic dietary advice based on a booklet edited by the French Institute for Health Promotion and Health Education [Institut National de Prévention et d'Education pour la Santé, INPES] (42). Sections from the booklet were read and briefly commented on by the dietitian with each participant during the 30 minute appointment. The booklet was then given to the participant, who was also informed of their initial PANDiet score. During the second and third dietetic appointments, participants in the control arm were asked if they had any questions about the booklet contents. If any medical questions came, women were referred to their antenatal care provider.

\section{Intervention arm (Method used to generate tailored dietary advice)}

During the first dietetic appointment, participants in the intervention arm also received the INPES booklet, which was read and commented on by the dietitian as for the control arm. But they also received three items of tailored dietary advice generated by the optimization software in order to improve the nutrient adequacy of their diets, as evaluated by the PANDiet score adapted for pregnancy $(34,35)$. The participants were informed of their initial PANDiet score at the start of the appointment and it was explained that each piece of dietary advice proposed by the software could optimally improve their initial score. The software included an algorithm that calculated the initial PANDiet score of each participant (using her dietary data), then implemented a stepwise dietary 
optimization model designed to improve the initial PANDiet score. For each piece of dietary advice, a participant could choose between three options proposed by the algorithm, two that best increased the PANDiet score by replacing a food item consumed in the initial diet with a food item from the same subgroup, and one that best increased the PANDiet score by modifying the amount consumed of a food item from the initial diet. These two types of dietary advice have already been described in depth and were chosen by considering a trade-off between their theoretical nutritional efficiency and their acceptability as evaluated during previous studies $(35,37)$.

The theoretical increase in the PANDiet score resulting from each option was communicated to the subject who then selected her preference. This choice was then implemented in the dietary record as a theoretical change that had been made, and the corresponding PANDiet score was calculated. This PANDiet score served as a reference to generate a new set of three options. At each step, tailored dietary advice should not decrease the initial EIEA and not increase it by more than $190 \mathrm{kcal}$ (i.e. the difference between the increase in energy requirements between the first and second trimester of pregnancy according to EFSA guidelines).

During the second and third dietetic appointments, participants in the intervention arm were asked if they had any questions about the booklet. They also received three more pieces of tailored dietary advice according to the process detailed above. In total, after the third dietetic appointment, participants in the intervention arm had received nine pieces of tailored dietary advice.

After each appointment, an email was sent to each participant that included the following information: her initial PANDiet score, the list of dietary advice that she had chosen during all previous appointments (i.e. three after the first dietetic appointment, six after the second one and nine after the third one), the theoretical increase in the PANDiet score procured by each change and an encouragement to read the booklet. A reminder was sent out one week after each appointment. To maintain the same intensity of interaction between the dietitian and participants in the intervention and control arms, participant in the control arm also received an email after each appointment and one week after that included the following information: her initial PANDiet score and an encouragement to read the booklet.

\section{Statistical analyses}

\section{Sample size calculation}

We computed the sample size required to obtain a statistically significant difference in the PANDiet score (primary outcome) between the two arms. A 5-point difference was defined as being nutritionally significant, based on our previous simulation studies $(34,35)$. This difference was the interquartile range in the population whose standard deviation was 7 points (34). Furthermore, in 
our simulations, the least theoretically efficient type of advice resulted in a $\sim 1$ point improvement per piece of advice, with a probability of intention to use it of $\sim 0.6$, so we reasoned that the nine dietary changes provided in the present study could be expected to lead to a $\sim 5$ point improvement in the PANDiet score (35). Considering a standard deviation of 7 points for the PANDiet score, a two-side power calculation required 32 participants per group, with an $80 \%(\beta=0.80)$ chance of demonstrating an effect of intervention on the PANDiet score at a 95\% confidence level $(\alpha=0.05)$. Allowing for a $20 \%$ dropout rate, we therefore sought to include 40 participants per arm.

\section{Descriptive and inferential statistics}

Descriptive statistics (mean, standard deviation, standard error of the mean and quartiles) were used to present continuous variables, and percentages were used for categorical variables. Differences between the arms were determined using Student t-tests for continuous variables and Fisher exact tests for categorical variables. The change in the PANDiet score and its associated subscores and the probabilities of adequacy for nutrient intakes were calculated as the final value minus the initial value. The primary outcome measure of the study was the change in the PANDiet score. In a first model, which addressed the pre-specified analysis, we tested the effect of the intervention using a ttest, while further models of analysis were secondary post-doc analyses. In a second model we added the initial PANDiet score as a covariate using ANCOVA. Control variables were included as well as the intervention and initial PANDiet scores in two additional models to test for possible prediction of the change in the score. The third model included the following control variables assessed at baseline: age, BMI, parity, household income per person, level of education and attention paid to the diet score (sum of attention paid to the diet before pregnancy and since the start of pregnancy assessed using five degree Likert scales). The fourth model also included the control variables assessed in the final questionnaires: attention paid to reading the booklet (4 levels: Reading each section of the booklet very carefully - Reading the booklet with variations in attention depending on the section - Not reading the entire booklet but only the summary sheet presented on the two last pages - Not reading the booklet at all) and the attention paid to the diet since the start of the study (assessed using a five degree Likert scale).

A secondary analysis was performed in the intervention arm to determine whether changes in the PANDiet score differed depending on the number of pieces of advice actually implemented in the diet (as declared by the participant in the final questionnaire) or the sum of the frequency of implementing advice (as evaluated by the participant in the final questionnaire using a 5-level scale for each point: the score was calculated by attributing $0.25 \mathrm{pt}$ per level from always $(1 \mathrm{pt})$ to never $(0 \mathrm{pt}))$. 
All analyses were performed using SAS 9.1.3 (SAS Institute Inc., Cary, NC). $P<0.05$ was considered to be statistically significant.

\section{Results}

Characteristics of participants

Of the 80 women included between September and November 2016, equal numbers $(n=40)$ were randomly assigned to each arm and 78 women (38 in the control arm and 40 in the intervention arm) completed the study (Figure 2). The mean age of participants was $31 \pm 3.6$ years (Table 1), about one third of whom (35\%) already had at least one child. More than half of the women (56\%) belonged to the "senior executive" socio-professional group and almost half (49\%) had a household income higher than $€ 5,000$ per month. Almost all the women (91\%) had pursued their education for at least two years after high school. At inclusion, the mean number of weeks of amenorrhea was 19 \pm 2.6 . A great majority of the women in our study (87\%) had paid specific attention to consuming a healthy and balanced diet before this pregnancy, and $82 \%$ declared they had paid more attention such consumption since the start of this pregnancy. No differences between the arms were observed regarding all these variables.

\section{Effect of tailored dietary advice on changes to the PANDiet score}

The mean initial PANDiet scores were $\sim 60$ points and similar in both arms (Table 2). The PANDiet increased significantly in the intervention arm $(+3.6 \pm 9.3$ points $)$ but remained unchanged in the control arm $(-0.3 \pm 7.3$ points $)$, and the change in the intervention arm differed from that in the control arm. No effect of the intervention was observed in the change of the sub-scores or probabilities of adequacy except for ALA (whose improvement was significantly higher in the intervention arm). The probabilities of adequacy for ALA, thiamin, folate and cholesterol intakes increased in the intervention arm. The initial and final probabilities of adequacy composing the PANDiet score, as well as their changes, are presented by arm in Online Supplemental Table 1. The mean initial EIEA values were $1878 \pm 426 \mathrm{kcal} / \mathrm{d}$ in the total population, with no difference between the arms, while the mean final EIEA values were $1850 \pm 400 \mathrm{kcal} / \mathrm{d}$, with no difference between the arms. No differences in the change of the EIEA was observed between the arms.

\section{Effect of the initial PANDiet score on the change in the PANDiet score}

The higher the initial PANDiet score, the less marked was the change in the PANDiet score $(\beta=-$ 0.49; $P<0.0001)$ and we found an interaction between the initial PANDiet score and the effect of the intervention. When stratifying according to quartiles of the initial PANDiet score, the improvement in the PANDiet score was greater in the first than in the last initial PANDiet quartile. 
After a median split, we found that in the population with a lower initial PANDiet score, the change in the PANDiet score was $0.70 \pm 8.02$ in the control arm and $7.32 \pm 7.41$ in the intervention arm, which was approximately twice the degree of effect found for the whole population (Figure 3).

\section{Analysis with control variables}

Using model 3, we found that as well as the intervention, the initial PANDiet score and the attention paid to the diet score had an effect on the change of the PANDiet score. When other control variables were added (determined at the end of the study), same results were found as well as some trends regarding the effects of the attention paid to reading the booklet and to the diet since the start of the study ( $P=0.08$ for both variables).

\section{Evaluation of tailored dietary counseling}

Fewer than $5 \%$ of women in each arm did not read the booklet at all after the first dietetic appointment. Participants in the control arm read the booklet more often than those in the intervention arm. Indeed, $29.0 \%$ of the women in the control arm declared that they read the booklet five times or more, whereas all women in the intervention arm read it four times or less. Table 3 summarizes the characteristics of the dietary advice chosen by women in the intervention arm (number by type, number of pieces of advice implemented in the diet and intensity of implementation of this advice). There was no difference between the number of pieces of dietary advice involving modifications to the amounts consumed $(4.4 \pm 1.7)$ and the number of dietary advice involving a substitution $(4.6 \pm 1.7)$ chosen by the participants. Women in the intervention arm largely reported having implemented the dietary advice (intensity of implementation: $5.7 \pm 1.4$ points out of 9). In the intervention arm, we found no effect of the number of pieces of advice effectively implemented in the diet or of the intensity of their implementation on the change in the PANDiet score.

\section{Discussion}

Tailored dietary counseling using a computer-based algorithm was more efficient than (pregnancyfocused) generic dietary counseling alone in improving the nutrient adequacy of the diet of French women in mid-pregnancy. An improvement in the nutrient adequacy of the diet was observed among participants who received generic plus tailored dietary counseling, whereas no such change was detected among those who only received generic dietary counseling based on a booklet. Furthermore, we found that the intervention improved the probabilities of adequacy for key nutrient intakes during pregnancy such as ALA and folate. 
Among interventions that offer dietary counseling to pregnant women, some have proved effective in improving diet quality during pregnancy (28-30). However, these interventions differ considerably in terms of the characteristics of the women concerned, the nature of dietary counseling and its objective (to improve pregnancy, maternal or infant outcomes). A majority of recent studies have focused on obese or overweight women or women with/or at-risk of gestational diabetes mellitus. Few studies have only included women with a normal pre-pregnancy BMI (28). Given this scarcity of data, the present study makes an important contribution to dietary counseling in healthy pregnant women.

In other groups of population, tailored nutrition interventions have proved to be effective, mainly because personalization made the advice more acceptable to the targeted population $(31,33,43)$. Thus the tailored dimension of our approach might have increased its acceptability and hence the effective implementation of dietary advice. The women were informed that the advice generated using the application was specific to their diet and would not have been proposed to another woman with a different diet. This may have enhanced their feelings of identification with the approach when compared to the generic advice. Furthermore, some existing behavior change techniques were used to improve the adherence of women to the intervention (44). Indeed, they were aware that each piece of advice was generated to optimize their own nutrient adequacy score evaluated using their declared diets and they were told about the theoretical benefits of each type of advice (in points) to improving their PANDiet score. This is referred to as "provide information on consequences of behavior to the individual" according to the CALO-RE taxonomy (44). Furthermore, the nine pieces of dietary advice were not chosen during the same session. The provision of three, two-week spaced sessions offered them an opportunity to gradually implement the advice in their diets. This is referred to as "set graded tasks" in the CALO-RE taxonomy. At the end of each session, the list of advice was reviewed with the dietitian and confirmed automatically by email to each participant. A reminder was also sent one week later (referred to as "goal setting (behavior)"). The second and third sessions also provided an opportunity to review these previously set goals with the participant (referred to as "prompt review of behavioral goals"). At the end of dietary follow-up, the final nutrient adequacy score was sent to all participants by email (referred to as "provide feedback on performance"). To ensure that the reception of tailored advice was the only difference between the two arms, the booklet content was discussed during each session with women of both groups. Furthermore, women in the control arm were also aware of their scores and knew they could use them as a measure of improvements made to their diet by the generic guidelines at the end of the follow-up. However, the tailored approach naturally enabled a more intensive use of behavior change techniques. 
Furthermore, few previous interventions had employed a computer-based tailored approach to improve the diet of women during pregnancy. During a randomized controlled trial, Jackson et al. used a computer-based video counseling program to provide advice on physical activity and diet to pregnant women of various BMIs (25). Diet-related advice was tailored to each woman's dietary habits and their motivation to change the behavior targeted by each piece of advice. Both dietary habits and motivation were assessed using questionnaires generated by the computer-based program, giving complete autonomy to the women. Within four weeks, the authors observed greater increases in the consumption of fruits and vegetables, fish, avocado and nuts and whole grain products, and more marked reductions in that of solid fats and fried foods in the intervention arm versus the control arm (25). Their study and ours concur regarding the view that tailoring associated with behavior change techniques could be a key determinant in improving diet quality during pregnancy. However, unlike ours, this study only focused on the consumption of certain food groups (and not on individual nutrient intakes) and dietary counseling only concerned four components of the diet (fruits and vegetables, "healthy" fats, whole grains and sugary foods). Until now, nutrient-based approaches to dietary counseling have only focused on one (45) or several nutrients (24,46-48), but mostly macronutrients. To our knowledge, even if some nutrients have been identified to be key during pregnancy, such as folate, DHA, or iron, there is no literature to define specific weights to those nutrients when setting PANDiet score parameters. Finally, the specific feature of our study and its findings was that it covered a large set of nutrients and generated tailored dietary advice using an algorithm to improve the overall nutrient adequacy during pregnancy. For this work, we chose a measure of dietary quality applied at the nutrient level, which is holistic and not specific to pregnancy, yet parametrized for pregnant women.

Overall, our tailored approach improved the nutrient adequacy of the diet of pregnant women during the study period, with particularly marked improvements among those with the lowest initial PANDiet scores. This result is of particular interest because it means that the benefits of intervention are greater in the women who need this most, which is a key characteristic of efficiency in public health nutrition. By contrast, it should be pointed out that in the intervention arm, only a few significant improvements were achieved when each probability of adequacy for nutrient intakes was considered separately. Accordingly, in the event of major deficiencies in specific nutrients deemed important for pregnancy, this approach should be associated with supplementation (49). However, when no major deficiencies are observed as in the case of our participants, our results indicate that this tailored approach could be valuable in improving the overall nutrient adequacy of the diet of pregnant women without resorting to multiple nutrient supplementation (50) which is often the case during pregnancy (14). The tailored approach would also be better than taking multiple supplements because, with a few dietary changes, it can improve 
overall nutritional status, avoid excessive intakes, address the problem of nutrients whose intake needs to be reduced and promotes healthier dietary practices that could be maintained after pregnancy.

\section{Limitations}

Two main limitations could be identified as affecting this study. The first concerned the dietary assessments. Dietary data were recorded online, as is already done in France (51), which enabled participants to benefit from complete autonomy and to automate the data collection process, but it restricted the number of days that could be recorded. Using three days of dietary record at the end of dietary follow-up limited the possibility to detect any effects of the number of pieces of dietary advice that were actually implemented in the diet. Furthermore, pregnancy is accompanied by many specific physiological changes that affect food intakes and choices, such as nausea, acid reflux or tiredness (36), which can result in important inter-individual and intra-individual (between days within a dietary record and between both final and initial dietary records) variations in food intake. Taken together, these two limitations were expected to cause an underestimation of the true effect of the intervention and reduce the statistical power of the study.

The second limitation concerned the socio-demographic characteristics of our population which was predominantly composed of women with high levels of education, household income and sociooccupational categories and living in an urban area. The implementation of dietary advice was therefore not limited by affordability or availability concerns. Furthermore, most of the women were already aware of the importance of good nutrition and had paid specific attention to consuming a healthy and balanced diet before their pregnancy, as shown by the relatively high initial PANDiet scores when compared to previous reports in French women $(34,35)$. Because we found that the intervention was more efficient in women with a lower nutrient adequacy, this limitation may also have led to an underestimation of the degree of effect that might be expected in a more general population. The effect size of the intervention nevertheless remained quantitatively important ( 0.5xSD in the overall population and $\sim$ SD in those with a lower initial score), particularly if it was attributed to just the three sets of three pieces of advice regarding three dietary items. Giving the findings obtained with this specific population, another study was performed in a more deprived area also in Paris with vulnerable pregnant women. Results obtained for populations whose socio-demographic characteristic differed could be compared.

\section{Perspectives}

Given the extent of demand related to dietary counseling during pregnancy $(36,37)$ confirmed by the very low attrition rate in this study, the findings of this study may encourage the proposal of 
dietary counseling as a regular process during antenatal care. Nevertheless, to deploy a tailored dietary approach during pregnancy at a larger scale, time and cost should be assessed. Furthermore, pregnant women have been known to express their interest in benefiting from tailored dietary advice (37), and the resulting higher compliance may be form of the success of this intervention in improving the overall nutrient adequacy of the diet. This lends credence to the idea that pregnancy is confirmed to be a teachable moment (52) in favor of adopting healthier behaviors. Therefore, it would be interesting to conduct a dietary intervention during pregnancy and to follow women after delivery to assess whether the healthier dietary behaviors that they adopted during the intervention are then maintained.

\section{Conclusion}

When accompanying generic dietary information, tailored dietary counseling using a computerbased algorithm was more efficient than the generic information alone in improving the nutrient adequacy of the diet of French women in mid-pregnancy, particularly among women with a lower initial diet quality.

\section{Acknowledgments}

The authors would like to thank the Notre-Dame de Bon Secours Maternity Clinic for hosting this study.

Financial support: this study received support in the form of a grant from Danone Nutricia Research and Blédina. Blédina was not involved in the design, conduct, analysis and interpretation of the study. Danone Nutricia Research did not have a decision-making role in the design, conduct, analysis and interpretation of the study; however, an employee of this company, co-author of this manuscript, who have some expertise in dietary advice participated in the discussions about some points of the design of the study (not on the content of the algorithm that provided dietary advice, including for the nutrients, the food groups/items, and the types of dietary advice that were considered). This employee was also involved in the conduct of the study by receiving regular information on the follow-up of the study and giving feedbacks, but was not in contact with the participants. This employee was not involved in the analysis and the interpretation of the results. There was no commercial interest in the frame of this study. The dietary advice tool that was tested in this study has never been the subject of any commercial valorization by the Blédina or Danone Nutricia Research. 
Conflict of interest: CM Bianchi, F Mariotti, C Journet, Y Stehr, H Beaussier, J Fournier, S Dervaux, D Cohen-Tanuggi, E Reulet, EO Verger, E Azria and JF Huneau declare no conflicts of interest. A Lluch is employed by Danone Nutricia Research.

\section{Authorship}

C.M.B, F.M, A.L, E.O.V, E.A and J.F.H designed the research and C.M.B, F.M, A.L, C.J, Y.S, H.B, J.F, R.E, E.O.V, F.M and J.F.H conducted the research. S.D and D.C.T provided essential material. C.M.B performed the statistical analysis, analyzed the data and wrote the first draft of the manuscript. All authors contributed to writing the manuscript and offered critical comments. C.M.B had primary responsibility for the final content. All authors read and approved the final manuscript.

\section{References}

1. Barker DJ. Fetal \& Infant Origins of Adult Diseases. London: British Medical Journal Publishing Group; 1992. 343 p.

2. Barker DJP. The developmental origins of adult disease. Eur J Epidemiol. 2003;18:733-6.

3. Hanson M. The birth and future health of DOHaD. J Dev Orig Health Dis. 2015;6:434-7.

4. Muhlhausler S, Ong ZY. The Fetal Origins of Obesity: Early Origins of Altered Food Intake. Endocr Metab Immune Disord - Drug Targets. 2011;11:189-97.

5. Pasternak Y, Aviram A, Poraz I, Hod M. Maternal nutrition and offspring's adulthood NCD's: a review. J Matern Fetal Neonatal Med. 2013;26:439-44.

6. Blumfield ML, Hure AJ, Macdonald-Wicks L, Smith R, Collins CE. Systematic review and meta-analysis of energy and macronutrient intakes during pregnancy in developed countries. Nutr Rev. 2012;70:322-36.

7. Blumfield ML, Hure AJ, Macdonald-Wicks L, Smith R, Collins CE. A systematic review and meta-analysis of micronutrient intakes during pregnancy in developed countries. Nutr Rev. 2013;71:118-32.

8. Goletzke J, Buyken AE, Louie JCY, Moses RG, Brand-Miller JC. Dietary micronutrient intake during pregnancy is a function of carbohydrate quality. Am J Clin Nutr. 2015;102:626-32.

9. Hauner H, Much D, Vollhardt C, Brunner S, Schmid D, Sedlmeier E-M, Heimberg E, Schuster T, Zimmermann A, Schneider K-TM, et al. Effect of reducing the n-6:n-3 long-chain PUFA ratio during pregnancy and lactation on infant adipose tissue growth within the first year of life: an open-label randomized controlled trial. Am J Clin Nutr. 2012;95:383-94. 
10. Bernard JY, Agostini MD, Forhan A, Lauzon-Guillain B de, Charles M-A, Heude B, Group the EM-CCS. The Dietary n6:n3 Fatty Acid Ratio during Pregnancy Is Inversely Associated with Child Neurodevelopment in the EDEN Mother-Child Cohort. J Nutr. 2013;143:1481-8.

11. Caldwell KL, Pan Y, Mortensen ME, Makhmudov A, Merrill L, Moye J. Iodine status in pregnant women in the National Children's Study and in U.S. women (15-44 years), National Health and Nutrition Examination Survey 2005-2010. Thyroid. 2013;23:927-37.

12. Rayman MP, Bath SC. The new emergence of iodine deficiency in the UK: consequences for child neurodevelopment. Ann Clin Biochem. 2015;52:705-8.

13. Drouillet P, Forhan A, De Lauzon-Guillain B, Thiébaugeorges O, Goua V, Magnin G, Schweitzer M, Kaminski M, Ducimetière P, Charles M-A. Maternal fatty acid intake and fetal growth: evidence for an association in overweight women. The "EDEN mother-child" cohort (study of pre- and early postnatal determinants of the child's development and health). Br J Nutr. 2009;101:583-91.

14. Pouchieu C, Lévy R, Faure C, Andreeva VA, Galan P, Hercberg S, Touvier M.

Socioeconomic, Lifestyle and Dietary Factors Associated with Dietary Supplement Use during Pregnancy. PLoS ONE. 2013;8:e70733.

15. Kadawathagedara M, Kersuzan C, Wagner S, Tichit C, Gojard S, Charles MA, Lioret S, de Lauzon-Guillain B. Adéquation des consommations alimentaires des femmes enceintes de l'étude ELFE aux recommandations du Programme national nutrition santé. Cah Nutr Diététique. 2017;52:78-88.

16. Caron P. Neurocognitive outcomes of children secondary to mild iodine deficiency in pregnant women. Ann Endocrinol. 2015;76:248-52.

17. Szwajcer EM, Hiddink GJ, Koelen MA, van Woerkum CMJ. Nutrition awareness and pregnancy: implications for the life course perspective. Eur J Obstet Gynecol Reprod Biol. 2007;135:58-64.

18. Szwajcer EM, Hiddink GJ, Koelen MA, van Woerkum CMJ. Nutrition-related informationseeking behaviours before and throughout the course of pregnancy: consequences for nutrition communication. Eur J Clin Nutr. 2005;59 Suppl 1:S57-65.

19. Whitaker KM, Wilcox S, Liu J, Blair SN, Pate RR. African American and White women's perceptions of weight gain, physical activity, and nutrition during pregnancy. Midwifery. 2016;34:211-20.

20. Goodrich K, Cregger M, Wilcox S, Liu J. A qualitative study of factors affecting pregnancy weight gain in African American women. Matern Child Health J. 2013;17:432-40.

21. Wennberg AL, Lundqvist A, Högberg U, Sandström H, Hamberg K. Women's experiences of dietary advice and dietary changes during pregnancy. Midwifery. 2013;29:1027-34.

22. Lucas $\mathrm{C}$, Charlton KE, Yeatman $\mathrm{H}$. Nutrition advice during pregnancy: do women receive it and can health professionals provide it? Matern Child Health J. 2014;18:2465-78.

23. Ferrari RM, Siega-Riz AM, Evenson KR, Moos M-K, Carrier KS. A qualitative study of women's perceptions of provider advice about diet and physical activity during pregnancy. Patient Educ Couns. 2013;91:372-7. 
24. Dodd JM, Cramp C, Sui Z, Yelland LN, Deussen AR, Grivell RM, Moran LJ, Crowther CA, Turnbull D, McPhee AJ, et al. The effects of antenatal dietary and lifestyle advice for women who are overweight or obese on maternal diet and physical activity: the LIMIT randomised trial. BMC Med. 2014;12:161.

25. Jackson RA, Stotland NE, Caughey AB, Gerbert B. Improving diet and exercise in pregnancy with Video Doctor counseling: a randomized trial. Patient Educ Couns. 2011;83:203-9.

26. Wolff S, Legarth J, Vangsgaard K, Toubro S, Astrup A. A randomized trial of the effects of dietary counseling on gestational weight gain and glucose metabolism in obese pregnant women. Int J Obes 2005. 2008;32:495-501.

27. Muktabhant B, Lawrie TA, Lumbiganon P, Laopaiboon M. Diet or exercise, or both, for preventing excessive weight gain in pregnancy. Cochrane Database Syst Rev. 2015;6:CD007145.

28. O'Brien CM, Grivell RM, Dodd JM. Systematic review of antenatal dietary and lifestyle interventions in women with a normal body mass index. Acta Obstet Gynecol Scand. 2016;95:259-69.

29. Gresham E, Bisquera A, Byles JE, Hure AJ. Effects of dietary interventions on pregnancy outcomes: a systematic review and meta-analysis. Matern Child Nutr. 2014;12:5-23.

30. Gresham E, Byles JE, Bisquera A, Hure AJ. Effects of dietary interventions on neonatal and infant outcomes: a systematic review and meta-analysis. Am J Clin Nutr. 2014;100:1298-321.

31. Brug J, Oenema A, Campbell M. Past, present, and future of computer-tailored nutrition education. Am J Clin Nutr. 2003;77:1028S-1034S.

32. Celis-Morales C, Lara J, Mathers JC. Personalising nutritional guidance for more effective behaviour change. Proc Nutr Soc. 2015;74:130-138.

33. Broekhuizen K, Kroeze W, van Poppel MNM, Oenema A, Brug J. A systematic review of randomized controlled trials on the effectiveness of computer-tailored physical activity and dietary behavior promotion programs: an update. Ann Behav Med Publ Soc Behav Med. 2012;44:259-86.

34. Bianchi CM, Mariotti F, Verger EO, Huneau J-F. Pregnancy Requires Major Changes in the Quality of the Diet for Nutritional Adequacy: Simulations in the French and the United States Populations. Cardoso MA, editor. PLOS ONE. 2016;11:e0149858.

35. Bianchi CM, Huneau J-F, Barbillon P, Lluch A, Egnell M, Fouillet H, Verger EO, Mariotti F. A clear trade-off exists between the theoretical efficiency and acceptability of dietary changes that improve nutrient adequacy during pregnancy in French women: combined data from simulated changes modeling and online assessment survey. PlosOne. 2018, April 11 ;13(4):e0194764.

36. Bianchi CM, Huneau J-F, Le Goff G, Verger EO, Mariotti F, Gurviez P. Concerns, attitudes, beliefs and information seeking practices with respect to nutrition-related issues: a qualitative study in French pregnant women. BMC Pregnancy Childbirth. 2016;16:306.

37. Bianchi CM, Mariotti F, Reulet E, Le Goff G, Lluch A, Verger EO, Huneau JF, Gurviez P. Perception of tailored dietary advice to improve the nutrient adequacy of the diet by French pregnant women : a mixed methods study. 2017; 
38. Hercberg S, Deheeger M, Prezio P. SU.VI.MAX Portions alimentaires manuel-photos pour l'estimation des quantités. Polytechnica. Paris: Polytechnica; 2002. 132 p.

39. Black A. Critical evaluation of energy intake using the Goldberg cut-off for energy intake:basal metabolic rate. A practical guide to its calculation, use and limitations. Int $\mathrm{J}$ Obes. 2000;24:1119-30.

40. Anses. Table Ciqual 2013 Composition nutritionnelle des aliments [Internet]. 2013 [cited 2014 Oct 31]. Available from: https://pro.anses.fr/TableCIQUAL/index.htm

41. AFSSA. Apports nutritionnels conseillés pour la population française, 3e éd. AFSSA : Editions Lavoisier. 3rd ed. Paris: Lavoisier; 2001. 650 p.

42. INPES. Le guide nutrition de la grossesse. Paris: Institut National de Prévention et d'Education pour la Santé; 2015. 40 p.

43. Brug J, Campbell M, van Assema P. The application and impact of computer-generated personalized nutrition education: a review of the literature. Patient Educ Couns. 1999;36:14556.

44. Michie S, Ashford S, Sniehotta FF, Dombrowski SU, Bishop A, French DP. A refined taxonomy of behaviour change techniques to help people change their physical activity and healthy eating behaviours: the CALO-RE taxonomy. Psychol Health. 2011;26:1479-98.

45. Clark J, Craig L, McNeill G, Smith N, Norrie J, Devereux G. A novel dietary intervention to optimize vitamin E intake of pregnant women to 15 mg/day. J Acad Nutr Diet. 2012;112:297301.

46. Khoury J, Henriksen T, Christophersen B, Tonstad S. Effect of a cholesterol-lowering diet on maternal, cord, and neonatal lipids, and pregnancy outcome: A randomized clinical trial. Am J Obstet Gynecol. 2005;193:1292-301.

47. Hawkins M, Chasan-Taber L, Marcus B, Stanek E, Braun B, Ciccolo J, Markenson G. Impact of an Exercise Intervention on Physical Activity During Pregnancy: The Behaviors Affecting Baby and You Study. Am J Public Health. 2014;104:e74-81.

48. Korpi-Hyövälti E, Schwab U, Laaksonen DE, Linjama H, Heinonen S, Niskanen L. Effect of intensive counselling on the quality of dietary fats in pregnant women at high risk of gestational diabetes mellitus. Br J Nutr. 2012;108:910-7.

49. Haider BA, Bhutta ZA. Multiple-micronutrient supplementation for women during pregnancy. Cochrane Database Syst Rev. 2017;4:CD004905.

50. Parisi F, Laoreti A, Cetin I. Multiple micronutrient needs in pregnancy in industrialized countries. Ann Nutr Metab. 2014;65:13-21.

51. Touvier M, Kesse-Guyot E, Méjean C, Pollet C, Malon A, Castetbon K, Hercberg S. Comparison between an interactive web-based self-administered $24 \mathrm{~h}$ dietary record and an interview by a dietitian for large-scale epidemiological studies. Br J Nutr. 2011;105:1055-64.

52. Phelan S. Pregnancy: a "teachable moment" for weight control and obesity prevention. Am J Obstet Gynecol. 2010;202:135.e1-8. 


\section{Tables}

Table 1. Characteristics of the women included in the analysis of the randomized controlled trial by $\operatorname{arm}^{1}$ (control arm: $n=38$; intervention arm: $\left.n=40\right)$ and for the total study population $(n=78)$.

\begin{tabular}{|c|c|c|c|}
\hline & $\begin{array}{l}\text { Control } \\
(n=38)\end{array}$ & $\begin{array}{l}\text { Intervention } \\
\qquad(\mathrm{n}=\mathbf{4 0})\end{array}$ & $\begin{array}{l}\text { Total } \\
(n=78)\end{array}$ \\
\hline $\operatorname{Age}^{2}$ (years) & $31.4 \pm 3.63$ & $31.5 \pm 3.69$ & $31.4 \pm 3.64$ \\
\hline $\begin{array}{l}\text { Number of children }^{3} \\
0 \\
1 \\
2 \\
3 \\
\end{array}$ & $\begin{array}{c}65.8 \%(25) \\
29.0 \%(11) \\
5.3 \%(2) \\
0.0 \%(0) \\
\end{array}$ & $\begin{array}{c}62.5 \%(25) \\
30.0 \%(12) \\
5.0 \%(2) \\
2.5 \%(1) \\
\end{array}$ & $\begin{array}{c}64.1 \%(50) \\
29.5 \%(23) \\
5.1 \%(4) \\
1.3 \%(1) \\
\end{array}$ \\
\hline $\begin{array}{l}\text { Socio-professional group } \\
\text { Craftsperson, Storekeeper } \\
\text { Senior executive } \\
\text { Intermediate profession } \\
\text { Employee } \\
\text { Student } \\
\text { Inactive } \\
\text { Other }\end{array}$ & $\begin{array}{l}5.3 \%(2) \\
60.5 \%(23) \\
10.5 \%(4) \\
15.8 \%(6) \\
0.0 \%(0) \\
5.3 \%(2) \\
1.3 \%(1)\end{array}$ & $\begin{array}{l}0.0 \%(0) \\
52.5 \%(21) \\
15.0 \%(6) \\
17.5 \%(7) \\
2.5 \%(1) \\
7.5 \%(3) \\
5.0 \%(1)\end{array}$ & $\begin{array}{l}2.6 \%(2) \\
56.4 \%(44) \\
12.8 \%(10) \\
16.7 \%(13) \\
1.3 \%(1) \\
6.4 \%(5) \\
3.9 \%(3)\end{array}$ \\
\hline $\begin{array}{l}\text { Highest diploma } \\
\text { PhD or MD } \\
\text { Master } \\
\text { Bachelor } \\
\text { Undergraduate (two years after high school) } \\
\text { High school diploma } \\
\text { Technical diploma (two years after middle school) } \\
\text { No diploma, middle school education }\end{array}$ & $\begin{array}{l}2.6 \%(1) \\
60.5 \%(23) \\
7.9 \%(3) \\
18.4 \%(7) \\
7.5 \%(3) \\
2.6 \%(1) \\
0.0 \%(0)\end{array}$ & $\begin{array}{l}2.5 \%(1) \\
67.5 \%(27) \\
10.0 \%(4) \\
12.5 \%(5) \\
2.5 \%(1) \\
2.5 \%(1) \\
2.5 \%(1)\end{array}$ & $\begin{array}{l}2.6 \%(2) \\
64.1 \%(50) \\
9.0 \%(7) \\
15.4 \%(12) \\
5.1 \%(4) \\
2.6 \%(2) \\
1.3 \%(1)\end{array}$ \\
\hline $\begin{array}{l}\text { Household monthly income } \text { ind }^{3,4} \\
<€ 500 \\
€ 2,000-2,999 \\
€ 3,000-3,999 \\
€ 4,000-4,999 \\
\geq € 5,000\end{array}$ & $\begin{array}{l}0.0 \%(0) \\
15.8 \%(6) \\
13.2 \%(5) \\
26.3 \%(10) \\
44.7 \%(17)\end{array}$ & $\begin{array}{l}2.5 \%(1) \\
12.5 \%(5) \\
12.5 \%(5) \\
20.0 \%(8) \\
52.5 \%(21)\end{array}$ & $\begin{array}{c}1.3 \%(1) \\
14.1 \%(11) \\
12.8 \%(10) \\
23.1 \%(18) \\
48.7 \%(38)\end{array}$ \\
\hline $\begin{array}{l}\text { Number of weeks of amenorrhea }^{2} \\
\text { Primiparous }^{3}\end{array}$ & $\begin{array}{l}18.8 \pm 2.51 \\
65.0 \%(25)\end{array}$ & $\begin{array}{l}19.1 \pm 2.76 \\
62.5 \%(25)\end{array}$ & $\begin{array}{l}19.0 \pm 2.63 \\
64.1 \%(50)\end{array}$ \\
\hline $\begin{array}{l}\text { Attention to a healthy diet } \\
\quad-\quad \text { Before this pregnancy } \\
\text { Totally agree } \\
\text { Agree } \\
\text { Neither agree nor disagree } \\
\text { Disagree } \\
\text { Totally disagree }\end{array}$ & $\begin{array}{l}31.6 \%(12) \\
57.9 \%(22) \\
10.5 \%(4) \\
0.0 \%(0) \\
0.0 \%(0)\end{array}$ & $\begin{array}{l}32.5 \%(13) \\
52.5 \%(21) \\
10.0 \%(4) \\
2.5 \%(1) \\
2.5 \%(1)\end{array}$ & $\begin{array}{c}32.1 \%(25) \\
55.1 \%(43) \\
10.3 \%(9) \\
1.3 \%(1) \\
1.3 \%(1)\end{array}$ \\
\hline $\begin{array}{l}\quad-\quad \text { Since the beginning of pregnancy }{ }^{\mathbf{3 , 6}} \\
\text { Totally agree } \\
\text { Agree } \\
\text { Neither agree nor disagree } \\
\text { Disagree } \\
\text { Totally disagree }\end{array}$ & $\begin{array}{l}47.4 \%(18) \\
31.6 \%(12) \\
13.2 \%(5) \\
5.3 \%(2) \\
2.6 \%(1)\end{array}$ & $\begin{array}{l}35.0 \%(14) \\
50.0 \%(20) \\
12.5 \%(5) \\
2.5 \%(1) \\
0.0 \%(0)\end{array}$ & $\begin{array}{c}41.0 \%(32) \\
41.0 \%(32) \\
12.8 \%(10) \\
3.9 \%(3) \\
1.3 \%(1)\end{array}$ \\
\hline
\end{tabular}

${ }^{1}$ There was no difference between the arms regarding all the variables presented, as assessed using either Student's ttests for continuous variables or Fisher's exact tests for categorical variables. $P>0.05$

${ }^{2}$ Values are mean \pm SD

${ }^{3}$ Values correspond to the percentage of participants presenting the characteristic described in the first column followed by the associated number of participants in parentheses.

${ }^{4}$ Only answers including at least one participant are presented in the table.

${ }^{5}$ The statement was as follows: "Before this pregnancy, I paid a specific attention to consuming a healthy and balanced diet"

${ }^{6}$ The statement was as follows: "Since the beginning of this pregnancy, I have paid more attention to consuming a healthy and balanced diet" 
Table 2. Initial and final PANDiet scores and associated sub-scores and their changes by arm (control arm: $n=38$; intervention arm: $n=40$ )

\begin{tabular}{|c|c|c|c|c|c|c|c|c|c|}
\hline & \multicolumn{4}{|c|}{ Control $(n=38)$} & \multicolumn{4}{|c|}{ Intervention $(n=40)$} & \multirow{2}{*}{$P^{4}$} \\
\hline & Initial $^{1}$ & Final $^{1}$ & Change $^{1,2}$ & $P^{3}$ & Initial $^{1}$ & Final $^{1}$ & Change $^{1,2}$ & $P^{3}$ & \\
\hline PANDiet & $60.4 \pm 7.3$ & $60.1 \pm 8.7$ & $-0.3 \pm 7.3$ & 0.77 & $60.3 \pm 7.3$ & $63.9 \pm 8.1$ & $3.6 \pm 9.3$ & 0.02 & 0.039 \\
\hline Adeq-S & $53.3 \pm 17.0$ & $51.6 \pm 16.6$ & $-1.7 \pm 17.0$ & 0.50 & $55.6 \pm 14.1$ & $58.9 \pm 15.9$ & $3.4 \pm 15.6$ & 0.15 & 0.18 \\
\hline Mod-S & $67.6 \pm 13.7$ & $68.6 \pm 13.5$ & $1.0 \pm 13.7$ & 0.69 & $65.0 \pm 12.3$ & $68.8 \pm 11.6$ & $3.9 \pm 12.4$ & 0.06 & 0.38 \\
\hline
\end{tabular}

${ }^{1}$ Values are mean \pm SD

${ }^{2}$ Difference between the values obtained from the final dietary record and the initial dietary record in each arm.

${ }^{3}$ Student's t-tests were performed to determine whether the mean changes within each group were different from 0.

${ }^{4}$ Student's T-tests were performed to determine whether the changes differed between arms.

Table 3. Numbers of pieces of advice chosen according to the type of advice and intensity of the implementation of dietary advice in the diet, among women in the intervention $\operatorname{arm}^{1}(n=40)$

\begin{tabular}{|l|c|}
\hline Number of pieces of advice chosen & \\
$-\quad$ Modification of amounts & $4.4 \pm 1.7$ \\
& $(0-8)$ \\
$-\quad$ Substitution & $4.6 \pm 1.7$ \\
& $(1-9)$ \\
$-\quad$ Actually implemented in the diet & $7.8 \pm 1.3$ \\
& $(5-9)$ \\
\hline Intensity of the implementation of advice & $5.7 \pm 1.4$ \\
\hline
\end{tabular}

All values are mean \pm SD on the first line, followed by range between brackets 


\section{Figure Legends}

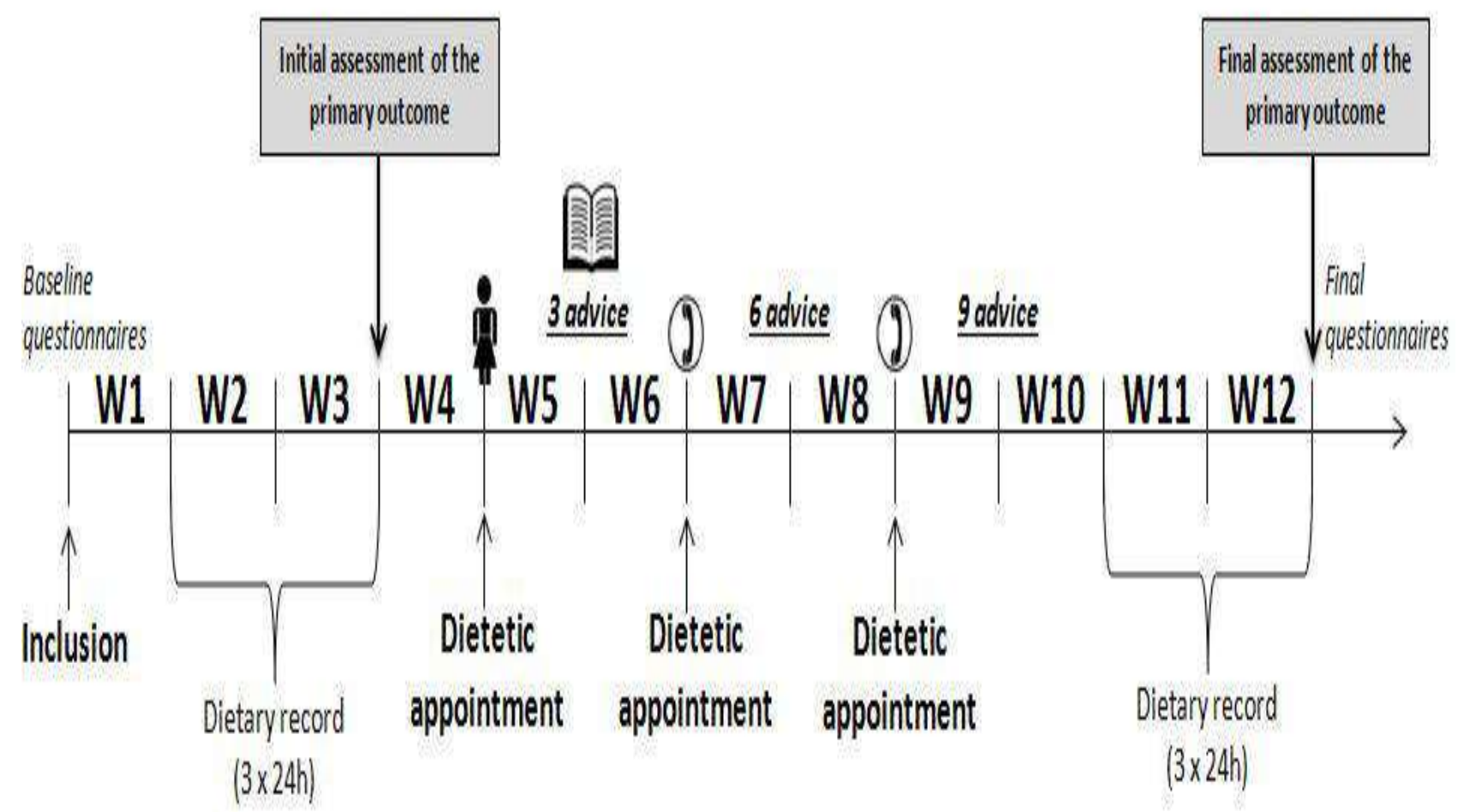

Figure 1. Timeline of 12-week dietetic follow-up for one participant. The pictogram showing a woman corresponds to a face-to-face dietetic appointment, whereas the pictogram showing a telephone corresponds to a dietetic appointment over the phone. The pictogram showing a book indicates the time point when the booklet was given to all participants.

Specificities for the intervention arm were presented in bold, italic and underlined characters.

$\mathrm{W}$, weeks. 


\section{Enrollment}

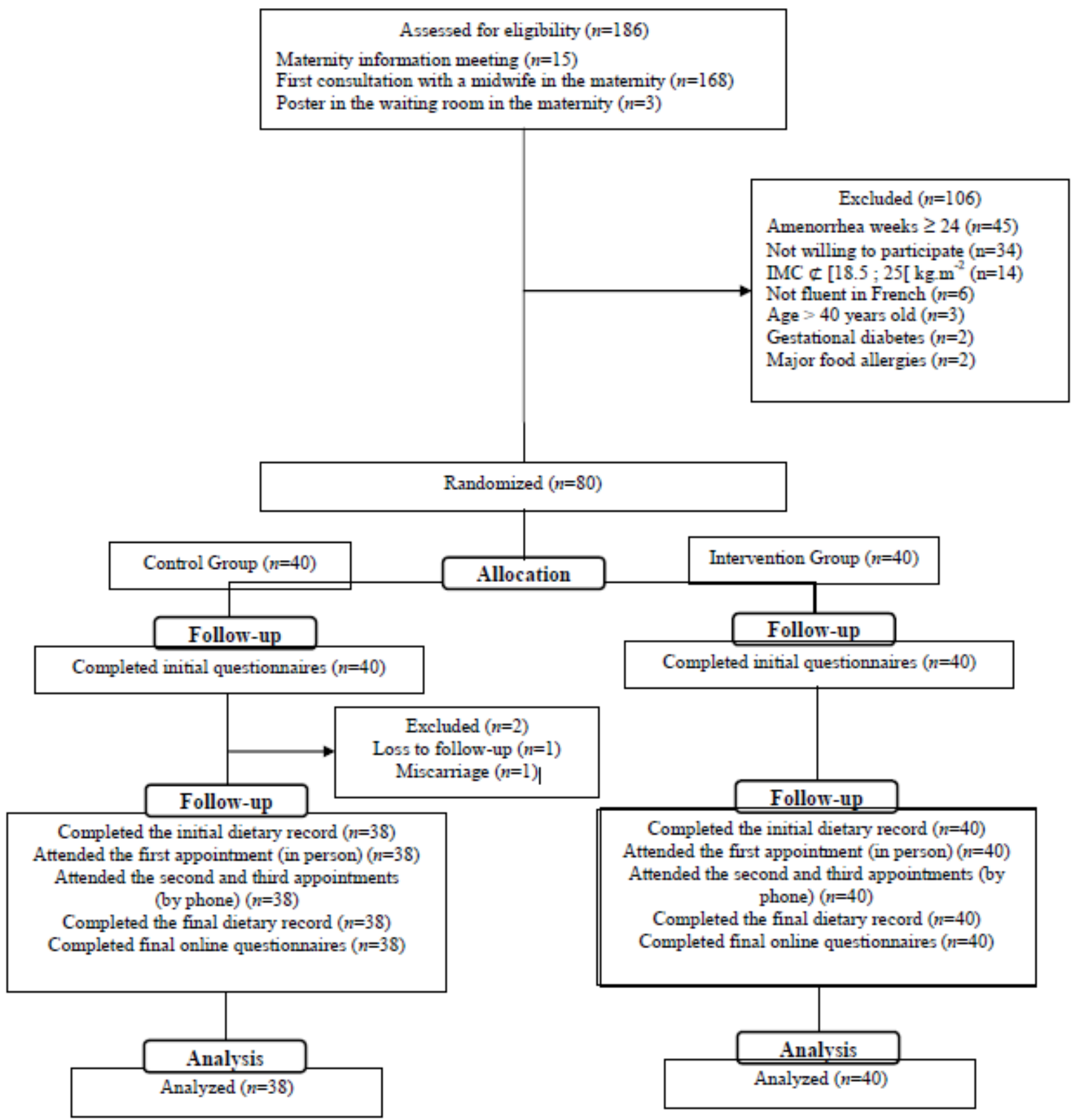

Figure 2. Flow diagram of a participant's progress through the study. 

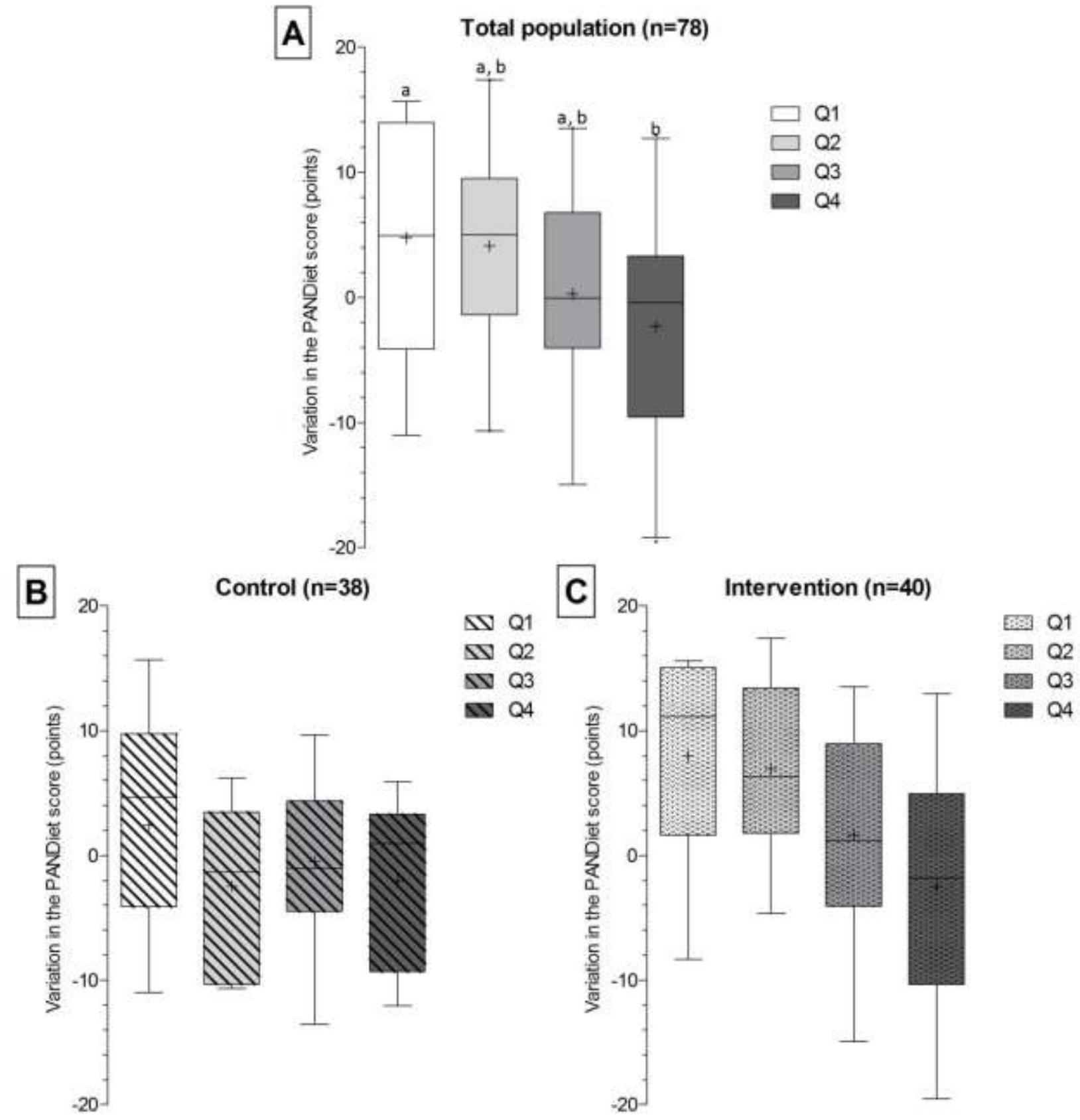

Figure 3. Boxplots of the change in PANDiet score by quartiles of the initial PANDiet score for the total population $(n=78$, panel $A)$, for the control arm $(n=38$, panel $B)$ and for the intervention arm ( $\mathrm{n}=40$, panel C). The white bars represent the first quartile of the PANDiet score, and the light grey bars represent the second quartile of the PANDiet score. The middle grey bars represent the third quartile of the PANDiet score, and the dark grey bars represent the fourth quartile of the PANDiet score. The middle line in the box plots shows the median, the cross in the box plots shows the mean, the bottom and top of the box are the 25th and 75th percentiles and the ends of the whiskers represent the 5th and 95th percentiles. This analysis was a secondary, post-hoc analysis.

Groups not showing the same superscript are significantly different within the same panel. Q1, first quartile. Q2, second quartile. Q3, third quartile. Q4, fourth quartile. 